$[\mathrm{n}=3]$; IV $[\mathrm{n}=3]$; mixed III/IV and V $[\mathrm{n}=2]$ ) and one patient had pure membranous LN. All children except two developed nephritic-nephrotic syndrome and AKI, and acute dialysis were initiated in 2 patients with TMA. At presentation, patients with renal vascular lesions had a significantly lower median eGFR (21.0, IQR 14.0-51.0 versus 88, IQR 48.5-107.5 ml/ $1.73 \mathrm{~m}^{2} / \mathrm{min} ; \mathrm{p}=0.011$ ) but similar degree of proteinuria $(3.1$, IQR 2.4-8.04 versus 2.5, IQR $1.3-5.1 \mathrm{mg} / \mathrm{mg}$ ) than those without such lesions.

Treatments were heterogeneous due to variable disease severity. In addition to pulse methylprednisolone, 6 and 3 patients received induction therapies with intravenous cyclophosphamide and mycophenolate mofetil, respectively. Three patients with severe AKI (LV $n=2$; TMA $n=1$; mean GFR $18.6 \mathrm{ml} / 1.73 \mathrm{~m}^{2} / \mathrm{min}$ ) responded to rituximab as add-on rescue therapies and eventually did not require acute dialysis. Therapeutic plasma exchange was performed in three patients with TMA as adjunctive therapies. Following induction, 6 patients attained complete remission and had normal GFR and no proteinuria at last follow-up. Three patients failed to respond and developed chronic kidney disease (CKD). One LV patient had poor adherence and repeated relapses leading to end stage kidney disease (ESKD) and received a kidney transplant. One patient with TMA and another with LV developed CKD II (GFR $74 \mathrm{ml} / 1.73 \mathrm{~m}^{2}$ / min) and CKD IV $\left(28 \mathrm{ml} / 1.73 \mathrm{~m}^{2} / \mathrm{min}\right)$, respectively. At the end of the study, more children with $\mathrm{LN}$ and renal vascular lesions developed either CKD or ESKD (3/6 [33.3\%] versus 4/48 [7.7\%], $\mathrm{p}=0.06$ ), although it did not reach statistical significance.

Conclusions Childhood-onset LN with renal vascular lesions had more severe presentation and may be associated with worse kidney outcomes. Identifying these lesions may have prognostic value and guide clinical management. Further largescale studies are required to define its role in the future LN classification.

\section{WEANING OFF INCUBATOR HUMIDIFICATION IN THE NEONATAL INTENSIVE CARE UNIT (NICU)- AN EVIDENCE UTILIZATION PROJECT}

Xia Wang, Soke Yee Lee, Tomomi Ogura, Sarasvati Rajoo. Singapore

\subsection{6/bmjpo-2021-RCPCH.170}

Background Infants born less than 32 weeks of gestation age are nursed in closed humidified incubator to minimise transepidermal water loss, prevent electrolytes imbalances and improve thermoregulation. The practice in the unit is these infants are cared for in closed incubators with $85 \%$ humidity for first 7 days of life, then from the second week onwards, gradually reducing the incubator humidity to $70 \%$, and from the fourth week, further decrease the humidity to $65 \%$. These infants are nursed in incubator with $65 \%$ humidity till they reached body weight of $1.7 \mathrm{~kg}$. The estimated length of stay in the humidified incubator for these infants is $1.5-3$ months. However, high or prolonged humidified environment is associate with Candida albicans, which has a reported mortality rate of $21-32 \%$ in very low birth weight infants. In 2015, two infants nursing in humidified incubator were detected having skin Candida albicans infection.
Objectives To minimise skin candida infection in infants requiring closed humidified incubator based on best available evidence.

Methods The length of stay in the humidified incubator and humidity setting were reviewed. In the new guideline, infant less than 32 weeks of gestation age is nursed in close incubator with humidity of $85 \%$ for the first 5 days of life. From the 6 th day onwards, to decrease incubator humidity by $5 \%$ daily, till it reaches the targeted discontinue setting of $60 \%$, which is 11 days of life. Five briefing sessions were conducted to all staff. Table of incubator humidity setting schedule are pasted in all incubators for easy reference. Staff compliance to new guideline was monitored daily. Project evaluation was by audits in April 2015 to June 2016.

Results The post-implementation and follow-up audit showed a $100 \%$ staff compliance. No candida infection was detected. The project has reduced the length of infant requiring humidified incubator to 10 days, which saved about SGD\$193.20 per infant per hospitalisation.

There were 244 infants (Year 2017: 52 infants, Year 2018: 68 infants, Year 2019: 59 infants, Year 2020: 65 infants) in total who were born less than 32 weeks of gestation age since project implementation to end of 2020. Further data showed there is no candida infection detected among these infants.

Conclusions The project provides clear humidity percentage guidelines for nurses to follow, and successfully achieved zero skin Candida infection on infants who are being nursed in closed incubator. The project provides clear humidity setting guidelines for nurses, and successfully achieved zero skin candida infection for infants who require close incubator.

\section{KNOTTED PERIPHERALLY INSERTED CENTRAL CATHETER IN A NEONATE}

Paula Tang, Yvonne Leung, Nicholas Chao, Michael Leung, Zita Hung, Kevin Fung. Hong Kong

\subsection{6/bmjpo-2021-RCPCH.171}

Background With the advance in technology and medical expertise, it has been increasingly common for clinicians to establish a secured vascular access in the hospitalized neonates. As the veins in preterm neonates are notoriously difficult to catheterize, clinicians are often left with limited options when a secured vascular access is clinically indicated for medications, fluids or intravenous nutrition.

Objectives Peripherally inserted central catheter (PICC) has the advantage of being easily available in most SCBU and NICU, does not require the input of anaesthetist or radiologist and they are often quite durable, lasting up to 3 to 4 weeks in a neonate with meticulous flushing and nursing care. While blood stream related infection, clot formation, malposition, occlusion and extravasation are commonly quoted PICC related complications in the literature, we report a case of tight knot formation of PICC during insertion in a neonate, subsequently requiring venotomy for retrieval.

Methods We report a case of a pre-term neonate with difficult PICC insertion and retrieval. Tight complex knotting of the PICC was confirmed by XRay, and the catheter was 